\title{
Methods in Science and Metaphysics ${ }^{1}$
}

\author{
Matt Farr and Milena Ivanova ${ }^{2}$
}

\begin{abstract}
While science is taken to differ from non-scientific activities in virtue of its methodology, metaphysics is usually defined in terms of its subject matter. However, many traditional questions of metaphysics are addressed in a variety of ways by science, making it difficult to demarcate metaphysics from science solely in terms of their subject matter. Are the methodologies of science and metaphysics sufficiently distinct to act as criteria of demarcation between the two? In this chapter we focus on several important overlaps in the methodologies used within science and metaphysics in order to argue that focusing solely on methodology is insufficient to offer a sharp demarcation between metaphysics and science, and consider the consequences of this for the wider relationship between science and metaphysics.
\end{abstract}

\section{What are science and metaphysics?}

Can we distinguish science from metaphysics? Traditionally, metaphysics is defined as the most general study of reality, concerned with the actual and possible, essences and potentialities, identities and priority relations. Science, on the other hand, is qualified as the study of the natural or physical or actual world. Already we face problems here: what is 'natural' and 'physical' if not just that which can be scientifically studied? Take any candidate for something non-physical or non-natural (e.g. ghosts, souls, spirits, and the like): if there were some way of reliably measuring it, then it would presumably be of relevance to science. Thoughts such of these raise scepticism as to whether metaphysics and science can be distinguished solely in terms of their subject matter. Although the subject matters of science and metaphysics may indeed overlap, it is reasonable to suppose that the disciplines may be distinguished in terms of their methodologies, particularly with respect to the empirical nature of the scientific method that has no obvious analogue in metaphysics. In the present chapter, we go further and make the case against a clear-cut methodological distinction between science and metaphysics. One might think that metaphysics is a purely conceptual, a priori, or 'armchair' discipline,

\footnotetext{
${ }^{1}$ To appear in James Miller and Ricki Bliss (eds), Routledge Handbook of Metametaphysics.

${ }^{2}$ Authors are listed in alphabetical order; this work is fully coauthored.
} 
whereas scientists are in the lab or the field, employing a distinctive empirical method that all and only sciences share, but the reality is much less clearly structured, with a variety of non-empirical methods shared by both metaphysicians and scientists in attempts to answer similar foundational questions about the world. In what follows we investigate different methodologies employed within science and metaphysics, both analytic and naturalistic, and argue that when it comes to methodology, there is a substantial overlap between science and metaphysics that undermines a sharp set of demarcation criteria between the two disciplines.

\subsection{What is science?}

Science is standardly understood to differ from non-scientific activities in terms of its method. Much of early $20^{\text {th }}$ century philosophy focused on identifying the scientific method and offering necessary and sufficient conditions for a discipline to constitute science as opposed to non-science or pseudo-science. The logical positivists employed a verificationist criterion of meaning, arguing that scientific statements are meaningful insofar as they are empirically verifiable, as opposed to metaphysical statements. Karl Popper (1963) argued that the scientific method is falsificationist as opposed to verificationist; although some scientific statements, such as universal generalisations, are not clearly verifiable through empirical means, it should be possible to falsify them through some empirical test. Thomas Kuhn (1970) offered a broader perspective on the demarcation problem and scientific methodology, taking genuine science to offer its own puzzle-solving tools that advance the scientific paradigm. Imre Lakatos (1977) appealed to the ability of scientific theories to make novel predictions to be what demarcates science from pseudo-science and to be a genuinely distinctive feature of progressive science. In attempting to resolve the demarcation problem and overcome objections raised against existing accounts, Paul Thagard (1978) offered a historical and social perspective on the question by focusing of the very practitioners of science. For Thagard, it is not only that pseudoscientific theories are less progressive than their competitors, but the very community of practitioners make little attempt at resolving problems endemic to their positions. On this account, demarcation is no longer an absolute matter but becomes contextual: evaluating the methods and approaches a community follows when addressing certain questions can make a theory scientific at one time and pseudoscientific at another. 
In the contemporary debate in philosophy of science, it is widely acknowledged that all such attempts to offer a set of necessary and sufficient conditions to demarcate science from non-science face a series of foundational problems. Many philosophers of science instead take science to adopt a plurality of methods, and that the relationship between science and non-science is resolved only when a more contextual approach is taken. What about metaphysics? Can we provide demarcation criteria for metaphysics, and is its methodology a helpful place to start looking?

\subsection{What is metaphysics?}

While once taken to constitute a single activity, science and metaphysics are now taken to be two very different disciplines. While science aims at making precise predictions about the physical world, metaphysics is taken to study questions of broader significance and generality. For instance, we turn to physics to predict where our planet will be with respect to the sun in a month's time; we turn to biology to understand the evolutionary difference between a sugar glider and a flying squirrel; we turn to metaphysics to address questions like "What is the essence of X?", "Are there universal properties?", "How do we understand actuality and possibility?", "What are the fundamental ontological categories?", etc.. While many questions of metaphysics are clearly outside the scope of science, some central questions, such as "Does time pass?" and "How does the mental relate to the physical?" are taken to fall under the study of both science and metaphysics, paving the way for what is commonly called naturalistic metaphysics. When it comes to questions of composition, finding a fundamental level to reality, or understanding time, modern science - whether physics, empirical psychology or biology - can contribute towards highly non-trivial answers. Naturalistic metaphysicians take science to provide the basis for their investigations, prescribing that we should read our metaphysics from our contemporary science, and answer questions about the fundamental nature of the world by appealing to fundamental physics (Ladyman and Ross (2007); Maudlin (2007); Ney (2012); French and McKenzie (2012; 2015); Morganti and Tahko (2017)). In this way, naturalistic metaphysics takes scientific theories to play a primary role in addressing many paradigm metaphysical questions.

On the other hand, analytic metaphysicians often see the relationship between science and metaphysics to be the other way around, with metaphysics being an autonomous area of study that determines the conceptual background that makes science possible. For instance, the metaphysician E.J. Lowe (2002, p. vi) holds that 
"metaphysics goes deeper than any merely empirical science, even physics, because it provides the very framework within which such sciences are conceived and related to one another." On the positive side, one can see an analogy here between, on the one hand, metaphysics and science and, on the other hand, pure and applied mathematics, with the metaphysician's job being to provide a kind of conceptual apparatus required for undertaking scientific study. On the negative side, one cannot neglect to take seriously the fact that metaphysics invariably has epistemic aims, such as establishing facts about the nature of how the universe operates (whether it be the function of laws of nature, or the nature of time, space, causation, or even existence itself), and it is highly controversial to hold that we can achieve such epistemic aims from the armchair, divorced from empirical findings.

We examine the metaphor of one discipline being 'prior' to the other in greater detail in section 3. Beforehand, in the next section, we shall see how the methodologies of science and metaphysics overlap though the shared use of non-empirical evaluative factors such as theory virtues, appeals to intuition, and the related use of modelling and inference to the best explanation.

\section{What are the methodologies of science and metaphysics?}

Traditionally, science was taken to proceed by observation, intervention through experimentation, and logical forms of inference in order to form and test hypotheses. This positivist picture of science can hardly be considered adequate in describing science today, since these considerations cannot be seen as either necessary or sufficient for science. Scientists often need to choose between competing explanations of the same observations and the only way they can do so is by employing non-empirical factors in their decision making, such as aesthetic considerations like simplicity and elegance. As Pierre Duhem (1954) pointed out, when it comes to choosing between competing (empirically equivalent) hypotheses on non-empirical grounds, one runs into a meta-problem: having to choose which aesthetic virtue is to be prioritised and how it is to be defined. What ultimately determines the choice is the scientist's intuition or 'good sense' (Ivanova 2010).

Furthermore, hypotheses are often accepted within the scientific community despite the unavailability of empirical confirmation. This point is best illustrated within contemporary high energy physics in which theories are entertained by the community despite either making predictions that cannot be tested due to 
technological constraints, or moreover failing to make specific predictions due to an overabundance of free variables within the theories, such as with multiverse cosmology and string theory (see Ellis \& Silk (2014)). Such problems have led some to propose non-empirical accounts of theory assessment and confirmation within physics (most notably Dawid (2013), though see the collection of papers in Dawid, Thébault \& Dardashti (2019)). Less controversially, physicists have historically placed a high degree of trust in scientific theories and hypotheses prior to empirical confirmation, such as with the Higgs mechanism prior to its famously complex empirical confirmation, and the atomic hypothesis prior to J.J. Thomson's cathode ray experiments. In each case, the restricted availability of empirical tests has prompted the use of alternative non-empirical grounds for supporting a theory. Beauty, elegance, simplicity, unity, and coherence with other frameworks are among considerations widely employed by scientists in this regard, with not only pragmatic weight placed upon these factors, but also epistemic significance, since such considerations are often taken to justify belief in the truth of theories. ${ }^{3}$

\subsection{Use of theory virtues}

Simplicity and parsimony have long been considered important elements in scientific reasoning. For instance, when discussing scientific methodology, Isaac Newton offered several Rules of Reasoning (or methods), with the first rule committing to parsimony:

Rule I. No more causes of natural things should be admitted than are both true and sufficient to explain their phenomena. As the philosophers say: Nature does nothing in vain, and more causes are in vain when fewer suffice. For nature is simple and does not include in the luxury of superfluous causes. (Newton 1999, P. 794)

Simplicity was also used by Poincaré (2001[1902]) in his defence of Euclidean geometry in light of the underdetermination of physical geometry by experience. In the context of the measurement problem of quantum mechanics, simplicity is often appealed to by defenders of the Everett interpretation insofar as unlike rival interpretations, such as collapse theories and the De Broglie-Bohm theory, it does not add extra mathematical structure or postulates to the orthodox quantum mechanics formalism. On the contrary, defenders of rival interpretations standardly dismiss the Everett interpretation on the grounds of failing to be ontologically

\footnotetext{
${ }^{3}$ For a historical exploration of such attitudes, see Chandrasekhar (1987), McAllister (1996), Ivanova (2017), and Hossenfelder (2018).
} 
parsimonious, since despite its lack of extra postulates, it is standardly taken to entail a branching multiverse with every possible measurement outcome actually occurring.

Theory virtues play a central methodological role within metaphysics, with competing pictures of the world standardly evaluated with reference to their simplicity, parsimony, and fit with other metaphysical theories. For example, in ontology, trope theorists (such as Campbell (1990)) take their theory to be preferable to rival theories on grounds of simplicity, since it holds that there exist only tropes. Objects are understood as ontologically derivative bundles of tropes, being nothing over and above their constituting properties. For a trope theorist, there is nothing more to a tree than its particular colour, shape, length, weight and mass. Contrary to the minimal ontology postulated by trope theorists, Armstrong (1993) defends a two category ontology, which postulates both particulars and universals, and Lowe (2006) defends a four category ontology, which holds there to be two fundamental categories of particulars (objects and tropes) and two categories of universals (kinds and properties). Both Armstrong and Lowe argue that the various theoretical virtues of their theories with respect to trope theories, such as their explanatory power, outweigh the perceived vice of their respective lack of simplicity.

The fact that both science and metaphysics employ theory virtues has been used as a reason to defend the legitimacy of metaphysics on methodological grounds as a means of establishing truths about the world. As L.A. Paul claims:

The theoretical desiderata we use to choose a theory include simplicity, explanatory power, fertility, elegance, etc., and are guides to overall explanatory power and support inference to the truth of the theory. [... I] the method can lead us to closer to the truth in science, it can lead us closer to the truth in metaphysics. (Paul 2012, p. 21)

On the other hand, Ladyman \& Ross (2007) argue that the similarity of methods here instead has the consequence of making metaphysics pseudoscientific:

Some metaphysicians have realized that they can imitate science by treating their kind of inquiry as the search for explanations[. ...] Taking the familiar explanatory virtues of unity, simplicity, non-ad hocness, and so on, they [...] argue with each other about whose particular metaphysical package scores highest on some loosely weighted vector of these virtues and requires the fewest unexplained explainers. On 
the basis of such reasoning, metaphysics is now often regarded as if it were a kind of autonomous special science, with its explananda furnished by the other sciences. (Ladyman \& Ross 2007, p. 17, emphasis added)

Ladyman (2012) is dismissive of such an approach at least in part because he sees theory virtues, in the spirit of Van Fraassen (1980), as being merely pragmatic devices for theory choice and not being concerned with truth per se, whereas metaphysics explicitly does aim at truth. An obvious rejoinder here is simply to hold that theory virtues are guides to truth, this being an attitude found not only within metaphysics but also in science. However, such a claim requires serious justification. Scientific realists have offered a number of arguments in defense of the idea that theory virtues can be indicators of truth, both a priori (Swinburne (1997)) and empirical arguments, often based on inferences from the history of science (McMullin (2009), Schindler (2018)), but these are not without problems. In particular, the a priori arguments often assume that nature itself is simple, making them circular, while empirical arguments suffer from being inconclusive, since inductive arguments from the history of science can be offered both in support of and against realism (Ivanova (forthcoming)).

\subsection{Use of intuitions}

A further salient point of overlap between the methodologies of science and metaphysics is the use of intuition. In addition to weighing competing theories with respect to theory virtues, metaphysicians also appeal heavily to their intuitions, with 'intuitiveness' commonly taken as a key desideratum in itself. For instance, adherents of the A-theory of time take it to be intuitive that time passes and that the distinction between past, present and future are mind-independent, with the B-theory's rejection of these claims being highly counter-intuitive. Scientists are not immune to talking about the role of their intuitions, their aesthetic sensibility, and their intuitive sense. As mentioned above, Duhem claims that what resolves theory choice in science often is the 'good sense' of the scientists. The mathematician and scientist Henri Poincaré similarly argues that scientists use their aesthetic sensibility as a "delicate sieve" to select "the most elegant and beautiful combinations" that the mind produces $(2001,397)$. But can we reasonably claim that intuitions play the same role in science as in metaphysics?

At first glance, intuitions appear to play a more central role in metaphysics than they do in science; after all, the scientist may appeal to experiment to test theories. Taking 
this viewpoint, French and McKenzie (2015) claim there is a key asymmetry between the use of intuition in science and metaphysics:

[I]n the scientific case, and arguably in [naturalistic metaphysics], the intuitions are functioning only as a starting point, a guide to what to try and justify by other means; by contrast in the [case of metaphysics] intuition itself has an essential justificatory role. (p. 29)

Though there is certainly a lack of symmetry between the two cases, we take the asymmetry to be far less clear-cut; in particular, intuition is demonstrably used as a tool of justification within science. Being distrustful towards intuitions as a philosopher of science is of course well placed, since the history of science shows intuitions to routinely run counter to scientific discovery. But can we really appeal to the traditional distinction between context of discovery and context of justification to claim that intuitions, when used in science, are only relevant in the former context, while in metaphysics they are also crucial in the latter? We think that such an attitude does not do justice to much of what happens in science.

Scientists, for better or worse, appeal to intuition not only to come up with hypotheses but also to justify their projects. They do so primarily in cases where there is insufficient empirical data to confirm or disconfirm the relevant theory. Poincaré claimed that the aesthetic intuition of scientists leads them to select the hypotheses that are most likely to be successful (Ivanova (2017)). Pierre Duhem argued that theory virtues cannot resolve theory choice, but rather it is the scientist's good sense that selects the most fruitful theories. The Nobel laureate Subrahmanyan Chandrasekhar held that aesthetic intuition can play an epistemic role in science, noting that "we have evidence [...] that a theory developed by a scientist, with an exceptionally well-developed aesthetic sensibility, can turn out to be true even if, at the time of its formulation, it appeared not to be so" $(1987,64){ }^{4}$ Aesthetic intuitions are commonly invoked in contemporary physics where theories are compared on grounds of naturalness, elegance and beauty (Green (1990), Chandrasekhar (1987), McAllister (1996), Ivanova (2017)). While it is doubtful whether our intuitions are any good at picking the right theory in advance of decisive empirical data, we cannot overlook the fact that scientists routinely employ intuitions to justify belief in a theory in the absence of empirical confirmation, and commonly take such intuitions to be a

\footnotetext{
${ }^{4}$ Chandrasekhar goes on: "[a]s Keats wrote a long time ago, "what the imagination seizes as beauty must be truth-whether it existed before or not" $(1987,64)$.
} 
guide to the likelihood of a theory's truth. In order to do justice to scientific practice, it is important to acknowledge that intuitions within science demonstrably go beyond the context of discovery.

It is, of course, important to distinguish the descriptive aspect here from the normative. It is a matter of fact that many scientists do use intuition as a means of justification, but should they do so? A traditional approach to addressing this question is to look at the history of science and establish the track record of intuitive thinking: have intuitions led us down the right track or mostly taken us astray? It is well known that much of contemporary science is highly counterintuitive, and that many revolutionary theories were initially poorly received due to their lack of fit with received wisdom, whether this being because of the entrenchment of certain concepts within science (such as quantum mechanics overturning the determinism of classical physics) or the social restriction of religious dogma (such as with the Copernican revolution and Darwin's theory of evolution by means of natural selection), but such examples are also exceptional cases that stand out for their conceptually revolutionary status; it does not follow that intuitions are of no use in the standard inferential practices central to the construction of theories and hypotheses. ${ }^{5}$ We take such track-record arguments to be inconclusive in resolving the normative question but take it that an independent justification is needed if any epistemic import is to be placed on one's intuition.

\subsection{Modeling, thought experiments and modes of inferences}

While the use of intuition and theory virtues are two very clear examples of the overlapping methodologies of science and metaphysics, they are not the only cases. Metaphysicians and scientists also use similar forms of inference and modeling, such as the use of the imagination and thought experiments, the use of inference to the best explanation, and modeling by abstraction and idealization. Both scientists and metaphysicians employ the imagination and the construction of thought experiments. Descartes' evil demon argument is a thought experiment that asks us to imagine what our experiences would be like were there no external world. Einstein's elevator thought experiment asks us to imagine what our experience would be like were we subjected to (a) uniform upward acceleration in an elevator or (b) the gravitational

\footnotetext{
${ }^{5}$ The idea that intuition plays a largely reliable role in making scientific inferences from the data and forming new hypotheses, is of course distinct from the evidently false claim that contemporary scientific theories give a picture of the world that is 'intuitive', taken to mean 'aligned with common sense'.
} 
force in a stationary elevator, in order to argue for the equivalence principle. Poincare's heat disk thought experiment (1902) asks us to imagine what our experience would be like were we living in a non-Euclidean disk exposed to nonuniform heat forces in order to argue for the underdetermination of geometry by experience and to establish the legitimacy of non-Euclidean geometries. The use of the imagination to generate hypotheses and justify them is common practice in both science and metaphysics. ${ }^{6}$

Furthermore, scientists as well as metaphysicians use inference to the best explanation (IBE) as a reliable form of reasoning. Scientists often explain the success of science by invoking IBE: an example is the predictive success of the atomic theory being explained by the fact that atoms are real, rather than fictitious, entities. Similarly, in metaphysics, Platonists invoke IBE to argue for the existence of abstract objects. The raspberries and strawberries in my breakfast bowl share something in common - they are all red. This fact cannot be explained, one may argue, unless we posit the property - redness - shared by these objects. Platonists argue that the best explanation for this resemblance is the existence of abstract objects. ${ }^{7}$

A further related point of methodological overlap is the use of abstraction and idealisation in the creation of models. Abstraction and idealisation are common in science; we omit and simplify the systems we study in order to model them and make predictions, and we also introduce elements to the system that are factually incorrect. For instance, when we try to predict economic behaviour we use models that make certain assumptions about agents, by simplifying the parameters involved in making choices and assuming certain patterns of behaviour that do not correctly describe individual human behaviour. Paul (2012) sees the job of the metaphysician to be continuous with that of the scientist insofar as each uses abstraction and idealisation to model different ways the world could be. The advantage that science holds over metaphysics is that experience will eliminate a good number of competitors, while the metaphysician needs to rely on theoretical desiderata to choose between these competing models.

\footnotetext{
${ }^{6}$ For further reading on the role of thought experiments in science, see Stuart (2016) and Salis \& Frigg (forthcoming).

${ }^{7}$ For a recent examination on the relationship between IBE in science and metaphysics, see Saatsi (2017).
} 
In summary, insofar as the practice of science routinely goes far beyond a strictly empirical method, there are many key points of overlap between the methodology of science and that of metaphysics.

\section{The relationship between science and metaphysics}

Since there is a substantial overlap of the methodologies of science and metaphysics, appealing to methodology itself fails to provide a sharp demarcation between science and metaphysics. As with Venn diagrams, partial overlap does not entail indistinguishability; the empirical aspects of scientific methodology are sufficient to make key aspects of science distinct from metaphysics. However there is an asymmetry here insofar as the central methodological tools of metaphysics are also central to science. In light of this methodological overlap, this section considers the relationship between science and metaphysics in more detail. Metaphysics and science are often spoken of using the metaphor of a disciplinary hierarchy. First, it is often held by analytic metaphysicians (such as in the above quoted passage of E.J. Lowe) that metaphysics is conceptually prior to science through its wider, more general scope, and its concern with the basic conceptual tools used by science. Second, many naturalistic metaphysicians take the opposing point of view, such that metaphysics is secondary to science through its need to 'keep up' or 'fit' with the latest received wisdom within science, with science-free metaphysics being deemed as either limited to analytic questions that are not about the world per se, or else being an illegitimate means of addressing questions that ought to be informed by science.

Can either of these offer a clear means of demarcating science from metaphysics? In this section, we see that the methodological overlap of science and metaphysics undercuts both avenues. First, the use of non-empirical methods within science includes the kind of self-reflective conceptual analysis common to metaphysics, undermining the idea that metaphysics is where concepts are formed and science where they are applied or tested. Second, science's usage of non-empirical methods poses a problem for those inclined towards positivist, falsificationist, or pragmatist criticisms of metaphysics as meaningless, pointless, or simply less legitimate than science due to its non-empirical nature. 


\subsection{Is metaphysics conceptually prior to science?}

Consider the characterisation offered by Paul (2012) that "metaphysics tries to tell us what laws, naturalness, properties, objects, persistence, and causal relations fundamentally are [...] and science tries to discover which entities there are or how these natures are exemplified" (p. 6), and Morganti and Tahko's (2017) related claim that metaphysics differs from science "in its greater generality and perhaps conceptual priority" and that "science represents at least an indirect 'testing ground' for metaphysical hypotheses, which thus get fleshed out, as it were, in the same process that employs them to provide an interpretation of our best scientific theories" (pp. 2560-1). This idea of a clear hierarchy of metaphysics as prior to science implies that science is not properly reflective on its own conceptual tools. To pick on just one example from many, we see that this is patently not the case with respect to causal relations in the case of physics. Much of the focus in quantum foundations since the publication of Bell's theorems $(1964,1976)$ has been to reflect on what causal structure is, in order to understand how the non-local quantum correlations are to be explained, and indeed what is ultimately sufficient to provide a causal explanation in general, with the ultimate goal of utilising quantum causal relations for new technologies. Similarly, the task of unifying general relativity and quantum mechanics has forced theoreticians to query what space and time are, and whether spatiotemporal relations are properly fundamental or derivative of some deeper concepts. We could suppose that the researcher employed by the physics department becomes a de facto metaphysician when carrying out such inquiries, but this would appear to spin the facts to fit an inappropriate demarcation criterion.

Rather, the general idea of the scientist requiring a set of well-defined metaphysical concepts and tools before getting to work overlooks the central task in foundational work within science, namely how to reflect upon and adjust key central theoretical terms, such as 'time', 'space' and 'simultaneity' (in the case of classical to relativistic physics), 'motion' (in the case of Aristotelian to Galilean physics), 'cause' and indeed even 'reality' and 'identity' (in the case of classical to quantum mechanics). These are all examples from physics, but that is not to pick out physics as a special case; one can find analogous examples throughout the sciences, such as 'living', and 'individual' in the case of biology. ${ }^{8}$ What we see routinely in the foundations of the sciences is a reflection of its practitioners upon the basic terms and concepts of inquiry in order to deal with well-confirmed anomalies in the data that fail to fit existing theory, or else

\footnotetext{
${ }^{8}$ For 'living', see Machery (2012); for 'individual', see Clarke (2010).
} 
deal with the problem of unifying accepted but prima facie incompatible theories (as with general relativity and quantum theory). As such, the conceptual priority of metaphysics is in danger of being overstated: it is central to scientific methodology to perform the kind of key conceptual analysis that metaphysicians aim to do, and this is invariably done in response to empirical findings.

This is not simply a point about the tendency of scientists to effectively 'do metaphysics', but more importantly concerns the epistemology of the kind of concepts used in science. Very often, physics (to pick again on a preferred example) requires us to consider possibilities not seriously entertained or explored by metaphysics, not least due to their unintuitiveness, such as the inertial-framedependence of simultaneity employed to understand the nature of light in special relativity, the lack of definiteness of properties of subatomic particles between measurements in order to explain their motion in quantum mechanics, and the use of non-Euclidean geometry to understand the gravitational force in general relativity. To pick on the latter point in a bit more detail: there was a long debate within philosophy concerning the status of Euclidean geometry given that some of its axioms, particularly the 'parallel postulate', fail to have the status of logical truths. On this point, Schopenhauer (1966 [1819], p. 130) held that "this truth is supposed to be too complicated to pass as self-evident, and therefore needs a proof; but no such proof can be produced, just because there is nothing more immediate," comparing the parallel postulate to the principle of contradiction. The fact that not only were consistent non-Euclidean geometries formulated but that they later proved useful in accounting for physical forces demonstrates that armchair reasoning about metaphysical possibilities can be less general and more restrictive than it may initially appear. It is often the empirical method of science, namely the existence of data that fails to fit existing theories, that forces us to take seriously possibilities that may not have been entertained as metaphysically possible, with this empirical grounding of conceptual analysis playing a key epistemic and motivational role in our understanding of central concepts within scientific theories. As such, when conceptual apparatus is most needed within science, namely in the foundations of new theories, the data often plays a crucial role in the development and application of concepts, and as such this undermines the idea of metaphysics alone putting in the 'background' needed for science to proceed. 


\subsection{Is metaphysics less legitimate than science?}

Finally, metaphysics has historically been demarcated from science through being a less legitimate discipline, whether it being due to having the hopeless epistemic aim of achieving synthetic a priori knowledge (as the empiricists would have it), of being concerned with meaningless pseudo-questions (as the positivists would have it), of being merely untestable (as the falsificationists would have it), or merely of having no practical relevance (as the pragmatists would have it). More recently, we see Ladyman and Ross (2007) hold that much of analytic metaphysics "fails to qualify as part of the enlightened pursuit of objective truth, and should be discontinued" (p. vii) through its failure to meet the science-first ideal of naturalistic metaphysics. But there are clearly questions studied by metaphysicians on which science doesn't reasonably bear, and which we have no particularly strong reason to regard as somehow constituting illegitimate intellectual inquiry. So long as the metaphysician does not have immodest epistemic aims and is not under the illusion that their inquiry is sufficient to uncover some deep, synthetic facts about the world, then it seems reasonable to consider scientifically-uninformed metaphysical inquiry into issues like the special composition problem (van Inwagen (1990)), or the conceivability of backwards causation (Dummett (1954; 1964), Black (1956)), as analogous to pure mathematics.

Such a picture is endorsed by French and McKenzie (2012), naturalistic metaphysicians who defend the legitimacy of analytic metaphysics, in part because the latter can and often does prove useful to the former. This way of seeing the role of metaphysics is analogous to other theoretical endeavours: just as mathematicians come up with different theoretical frameworks, some of which can find their application in science (non-Euclidean geometries, knot theory), such might be the fate of many projects of analytic metaphysics, making them legitimate as potential scientific tools. Of course, much of the subject matter of this kind of metaphysics may be of little to no intrinsic interest to the scientist, the philosopher of science, nor indeed the naturalistic metaphysician, but this lack of intrinsic interest does not amount to a lack of legitimacy of analytic metaphysics. ${ }^{9}$

Potential usefulness, however, is a low bar. Though legitimate, many of the questions tackled in the field of analytic metaphysics may be reasonably deemed 'pointless', as

\footnotetext{
${ }^{9}$ Indeed, Ladyman (2012) acknowledges this epistemically modest picture of metaphysics as not intrinsically worthless, but rather as less sure-footed than other a priori disciplines such as pure mathematics or logic due to the metaphysics' lack of an analogous notion of proof.
} 
Ladyman and Ross (pp. 29-30) note, in the spirit of the pragmatist C.S. Peirce, on the grounds that they venture far outside what can in principle be tested. In contrast, scientific hypotheses, in the spirit of Popper, are standardly characterised as inprinciple testable, or at least are sufficiently connected to the concept of experimental testing, and as such are not pointless. Can we then suppose that this is a key mark of discernibility between metaphysics and science; that the kinds of questions asked by the latter are not pointless? Ultimately, the methodological overlaps between science and metaphysics we have highlighted, specifically the widespread usage of non-empirical methodologies in science, once more undercut this putative means of demarcation. This turns out to be a key issue in the case of socalled 'post-empirical' science, namely those research programs of theoretical physics that fail to make empirically testable predictions, with string theory and multiverse cosmology being prime examples (Ellis \& Silk, 2014). A central question in this regard is whether post-empirical science deserves its name: has theoretical physics reached the point at which large parts of it no longer constitute science at all? What exactly is the alternative: to dismiss such research as non-scientific, or as merely pointless, speculative science, or simply as metaphysics? There is the significant worry here that one is merely left playing with semantics. What is relevant is that empiricallydriven scientific discourse invariably has and does venture outside what can ultimately be tested, that non-empirical methods are for that reason a key tool within science, and hence that scientific methodology can overlap significantly with that of metaphysics, making it hard to delegitimise metaphysics on grounds of methodology without also condemning much of science. ${ }^{10}$

\section{Bibliography}

Armstrong, D. M. (1993) A World of State of Affairs, Philosophical Perspectives, Vol. 7, Language and Logic, pp. 429-440

Bell, J. S. (1964) On the Einstein-Podolsky-Rosen paradox. Physics, 1(RX-1376), 195200.

Bell, J. S. (1976) The Theory of Local Beables. Epistemological Letters: March 1976, 11-24, reprinted as chapter 7 of Bell's 1987 Speakable and Unspeakable in Quantum Mechanics, Cambridge: Cambridge University Press

Bird, A. (2007) Nature's Metaphysics. Oxford University Press.

Black, M. (1956) Why Cannot an Effect Precede Its Cause? Analysis 16.3: 49-58.

\footnotetext{
${ }^{10}$ The authors thank Steven French for helpful comments. This paper was written during the quiet hours, amidst the haze of new parenthood; we dedicate it to our daughter, Cailyn.
} 
Campbell, K. (1990) Abstract Particulars, Oxford: Blackwell

Chandrasekhar, S. (1987) Truth and Beauty: Aesthetics and Motivation in Science. The University of Chicago Press

Clarke, E. (2010). The problem of biological individuality. Biological Theory, 5(4), 312325.

Dawid, R. (2013) String Theory and the Scientific Method. Cambridge University Press.

Dawid, R., Thébault, K., \& Dardashti, R. (Eds.). (2018). Why Trust a Theory?: Epistemology of Fundamental Physics. Cambridge University Press.

Duhem, P. (1954[1906]) The Aim and Structure of Physical Theory. Princeton: Princeton University Press.

Dummett, M. (1954) Can an Effect Precede Its Cause? Proceedings of the Aristotelian Society 28: 27-44.

Dummett, M. (1964). Bringing about the past. The Philosophical Review, 73(3), 338359.

Ellis, G., \& Silk, J. (2014). Scientific method: Defend the integrity of physics. Nature News, 516(7531), 321.

French, S., \& McKenzie, K. (2012). Thinking outside the (tool)box: Towards a more productive engagement between metaphysics and philosophy of physics. The European Journal of Analytic Philosophy, 8, 42-59.

French, S., \& McKenzie, K. (2015). Rethinking outside the toolbox: Reflecting again on the relationship between philosophy of science and metaphysics. In T. Bigaj \& C. Wuthrich (Eds.), Metaphysics in contemporary physics. Poznan Studies in the Philosophy of the Sciences and the Humanities (pp. 145-174). Amsterdam: Rodopi.

Greene, B. (1999) The Elegant Universe. W.W. Norton and Company.

Hossenfelder, S. (2018) Lost in Math: How beauty Leads Physics Astray, Basic Books

Ivanova, M. (2010). Pierre Duhem's good sense as a guide to theory choice. Studies in the History and Philosophy of Science, 41: 58-64.

Ivanova, M. (2017) Aesthetic Values in Science, Philosophy Compass, Vol. 12, DOI: 10.1111/phc3.12433

Ivanova, M. (forthcoming) Beauty, Truth and Understanding, in Ivanova and French (eds.), The Aesthetics of Science: Beauty, Imagination and Understanding, Routledge

Kuhn, T. (1970) Logic of Discovery or Psychology or Research? In Imre Lakatos and Alan Musgrave (eds.) Criticism and the Growth of Knowledge, Cambridge University Press, p. 4-10 
Ladyman, J. (2007) Does Physics Answer Metaphysical Questions, Royal Institute of Philosophy Supplements, Vol. 61, 179-201

Ladyman, J. and D. Ross (with D. Spurrett and J. Collier). 2007. Every Thing Must Go. Oxford: Oxford University Press

Lakatos, I. (1977) Philosophical Papers, vol. 1, Cambridge University Press

Lowe, E.J. (2006) The Four-Category Ontology, Oxford University Press

Machery, E. (2012). Why I stopped worrying about the definition of life... and why you should as well. Synthese, 185(1), 145-164.

McAllister (1996) Beauty and Revolution in Science. Ithaca, NY: Cornell University Press.

McMullin, E. (2009) The Virtue of a Perfect Theory, in The Routledge Companion to Philosophy of Science, Martin Curd and Stathis Psillos (eds.), Routledge

Morganti, M. and Tahko, T.E. 2017. Moderately Naturalistic Metaphysics. Synthese 194 (7): 2557-2580.

Newton, I. (1999[1687]) The Principia: Mathematical Principles of Natural Philosophy. I.B. Cohen and A. Whitman (trans) Berkeley, CA: University of California Press

Ney, A. (2012). Neo-positivist metaphysics. Philosophical Studies, 160(1), 53-78.

Popper, K. (1963) Conjectures and Refutations. London: Routledge and Kegan Paul

Paul, L.A. (2012) Metaphysics as Modeling: The Handmaiden's Tale, Philosophical Studies 160 (1): 1-29.

Poincaré, H. (2001 [1902]) Science and Hypothesis: Essential Writings of Henri Poincaré. ed. Stephen Gould, New York: Modern Library.

Saatsi, J. (2017) Explanation and explanationism in science and metaphysics. In Slater \& Yudell (eds.) Metaphysics and the Philosophy of Science: New Essays. Oxford University Press.

Salis, F. , \& Frigg, R. (forthcoming). Capturing the scientific imagination. In P. GodfreySmith \& A. Levy (Eds.), The Scientific Imagination. Oxford University Press

Schindler, S. (2018) Theoretical Virtues in Science: Uncovering Reality Through Theory, Cambridge University Press

Schopenhauer, A. (1966 [1819]) The World as Will and Representation, Vol II, Trans. E.F.J. Payne, Dover Publishing.

Stuart, M. T. (2016). Taming theory with thought experiments: Understanding and scientific progress. Studies in History and Philosophy of Science Part A, 58, 24-33

Swinburne R. (1997) Simplicity as Evidence of Truth, Milwaukee, WI: Marquette University Press 
Thagard, P. (2013[1978]) Why Astrology is a Pseudoscience, in Martin Curd, J.A.Cover and Christopher Pincock (eds.), Philosophy of Science, W.W. Norton and Co. $2^{\text {nd }}$ ed.

Van Inwagen, P. (1990) Material Beings, Ithaca: Cornell. 